\title{
UWB Differentially-fed Circular Monopole Antenna with Stable Radiation Pattern
}

\author{
Eva Antonino-Daviu ${ }^{1}$, Marko Sonkki ${ }^{2}$, Miguel Ferrando-Bataller ${ }^{1}$, Erkki Salonen ${ }^{2}$ \\ ${ }^{1}$ Institute of Telecommunications and Multimedia Applications (iTEAM), Edificio 8G, Universitat Politècnica de València, \\ Camino de Vera s/n, 46022, Valencia, Spain. \\ 2 Centre for Wireless Communications (CWC), P.O. Box 4500, 90014 University of Oulu, Finland.
}

\begin{abstract}
A small UWB circular monopole antenna with a differential feed is presented in this paper, as a solution to increase the stability of the radiation pattern of a single-fed circular monopole antenna. A detailed characteristic modes analysis is presented in order to support the selection of the feeding mechanism. Simulated active-S parameter is shown for the proposed antenna, showing a good matching for the UWB frequency range. Normalized surface current distributions are presented, and 3D radiation patterns are compared to a reference monopole. The antenna structure shows more stable and omnidirectional radiation patterns over the studied frequency range compared to a same sized single-fed reference monopole.
\end{abstract}

Index Terms - Differentially-fed Antenna, Planar Monopole Antenna, Radiation Pattern Stability, Ultrawideband (UWB) Antenna, Small Antenna.

\section{INTRODUCTION}

During the last decade, planar monopole antennas have shown great interest for UWB applications, since they present very attractive properties such us low profile, large impedance bandwidth, omnidirectional radiation pattern, low cost and easy construction [1].

Extensive investigations considering different shape geometries (square, rectangular, triangular, circular, elliptical, and so on) and/or feeding mechanism (double-fed, stepped or asymmetrical fed) have been carried out during these years, using $3 \mathrm{D}$ or $2 \mathrm{D}$ (coplanar) configuration for the monopole mounting [1]-[3].

Moreover, other similar antennas have been proposed latterly for UWB applications. These are printed wide slot antennas, which consist of a wide slot with some geometrical shape, etched in a finite ground plane and fed with a microstrip of coplanar waveguide (CPW) microstrip line terminated in a shaped stub [4]-[6]. Many designs have been proposed with different shapes geometries, showing very good behavior for UWB applications. Some experimental design guidelines have also been proposed for this kind of antennas [4].

Recently, some differentially driven printed wide-slot antennas have been reported, which provide UWB performance using a differential feeding mechanism, which eases antenna integration with differential wireless communications systems, providing better radiation pattern stability [7]-[9].
This paper presents a differentially excited monopole antenna with two antenna feeding ports, for UWB applications. The antenna is based on that presented in [10], which covered lower frequencies and where a detailed parametric study was made in order to investigate the influence of different geometrical parameters on the antenna performance.

The objective of the paper is to provide a design which increases the stability of the radiation pattern of a circular planar monopole (which usually presents a single port). An analysis based on characteristic modes (CM) will be performed, in order to support the selection of the feeding mechanism.

\section{ANTENNA STRUCTURE}

Fig. 1 presents the geometry of the proposed antenna with the coordinate system. As can be observed, the antenna consists of a circular planar monopole with two microstrip feeding lines inserted at the top and bottom of the planar structure. Small rectangular ground planes are inserted under the two feeding lines. The width of feeding lines is $2 \mathrm{~mm}$, which is not exactly $50 \Omega$ as the mutual coupling affects to the active impedance matching.

The antenna uses an air dielectric substrate $\left(\varepsilon_{\mathrm{r}}=1\right)$ of thickness $0.6 \mathrm{~mm}$. The antenna is fed by two $50 \Omega$ discrete ports with the same amplitude but opposite phase, in order to create a differentially-driven antenna. Total dimensions for the proposed antenna are shown in Table I.

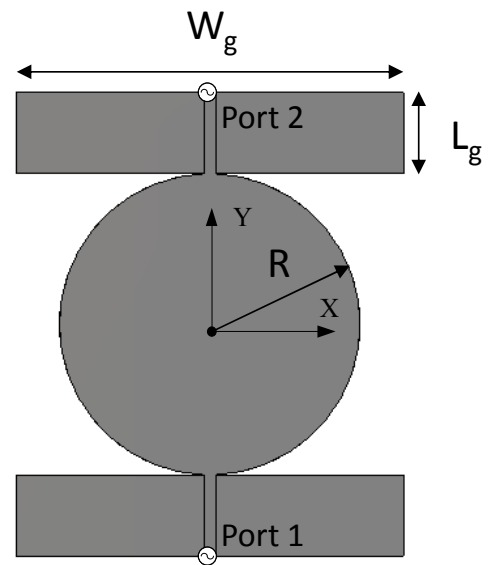

Fig. 1. Geometry of the proposed antenna with coordinate system. 
TABLE I. DimenSiONS of THE DifFERENTIALly-DRIVEN CIRCUlaR MONOPOLE

\begin{tabular}{|l|c|c|c|}
\hline Parameter & $W_{g}(\mathrm{~mm})$ & $L_{g}(\mathrm{~mm})$ & $R(\mathrm{~mm})$ \\
\hline Value & 26 & 5,4 & 10 \\
\hline
\end{tabular}

By means of the feeding configuration, vertical current distribution is reinforced in the structure. This method allows more stable radiation pattern than other current distributions, which may disturb the radiation pattern and polarization properties. In the next section a characteristic mode (CM) analysis will be performed in order to understand the physical radiating behavior of the antenna.

\section{CHARACTERISTIC MODES ANALYSIS}

In this section, the Theory of Characteristic Modes will be applied to study the radiating behavior of the proposed antenna.

\section{A. Characteristic Modes of a Circular Plate}

In Fig. 2, the current distribution of the first six $\mathrm{CM}$ of a circular plate of radius $\mathrm{R}=10 \mathrm{~mm}$ are shown. Characteristic angle variation with frequency is shown in Fig. 3 for the same modes.

As observed, eigenvector $J_{0}$ presents an inductive behaviour due to its current forming a closed loop over the plate. Eigenvectors $J_{l}$ and $J_{l}$ ' are degenerated modes presenting horizontal and vertical currents, while higher order modes $J_{2}, J_{2}$ ' and $J_{3}$ present increasing nulls along the perimeter of the plate.

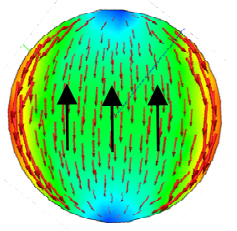

$J_{1}$

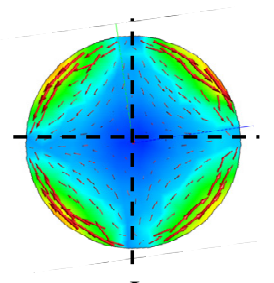

$J_{2}$

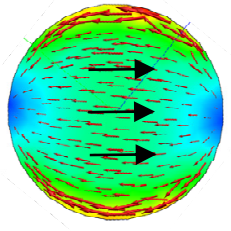

$J_{1}^{\prime}$

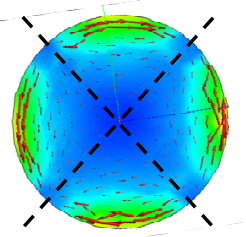

$J_{2}$,
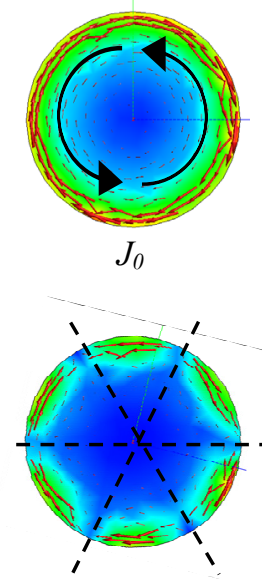

$J_{3}$
Fig. 2. Surface current distribution of the first six CM for a circular plate of radius $\mathrm{R}=10 \mathrm{~mm}$. Black arrows represent the direction of the current flow, whereas dashed lines denote nulls in the current distribution.

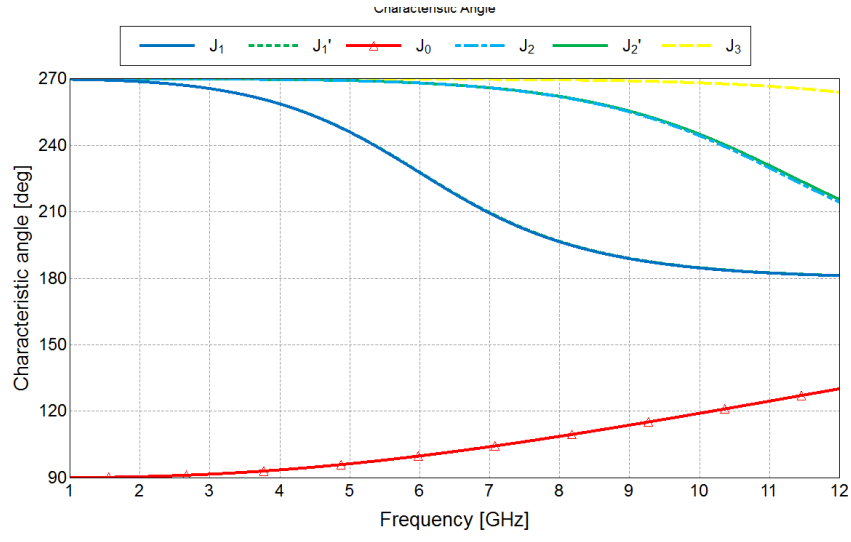

Fig. 3. Characteristic angle versus frequency for a circular plate of $\mathrm{R}=10$ $\mathrm{mm}$.

As presented recently in [11], a modal parameter can be used to assess the stability with frequency of the radiation pattern of each CM. This parameter, called Modal Pattern Stability Factor (MPSF), shows that mode $J_{l}$ of a circular plate exhibits a radiation pattern with increased stability when compared to other geometries such as square, or square with truncated corners, for vertical polarization [11].

Therefore, the aim is to excite mode $J_{l}$ in the circular plate in order to exhibit increased stability in the radiation pattern. With that purpose, two ports located at the top and bottom of the circular plate are used with $180^{\circ}$ phase shift (differential feeding), in order to force excitation of mode $J_{l}$ and prevent the excitation of other modes.

\section{B. Characteristic Modes of a Circular Plate with Two Ground Planes}

In order to differentially excite the circular plate at two points, two microstrip ports are used, as shown in Fig.1. In this section, a new CM analysis will be performed for the whole structure (circular plate with two rectangular plates acting as ground planes).

Fig. 4 shows the current distribution for the first nine $\mathrm{CM}$ of the differentially-fed antenna. Fig. 5 presents the characteristic angle variation with frequency associated to previous modes and, as observed, the number of CM within the analyzed frequency range increases with respect to those shown in Fig. 3 due to the combination of the CM of the circular plate with those of the ground planes. Moreover, as observed in Fig. 5, most of the modes decrease their resonant frequency due to the presence of the rectangular ground planes.

$\mathrm{CM}$ are independent of the excitation. Thus, if a differential feeding mechanism is used at the two ports, then modes with vertical current distribution at the circular plate will be excited. Therefore, it is expected that only modes $J_{l}$ and $J_{7}$ will be excited in the structure when using a differential feeding excitation. 


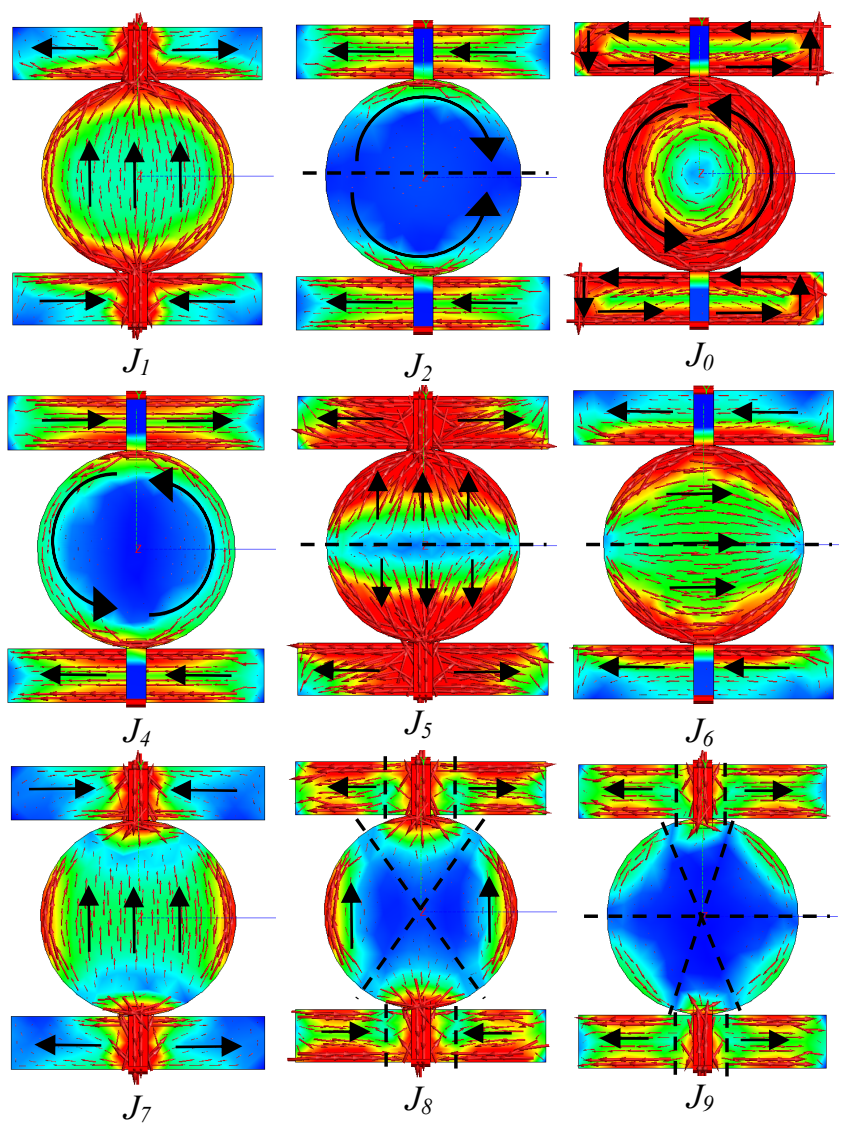

Fig. 4. Surface current distribution of the first nine $\mathrm{CM}$ of the proposed antenna. Black arrows represent the direction of the current flow, whereas dashed lines denote nulls in the current distribution.

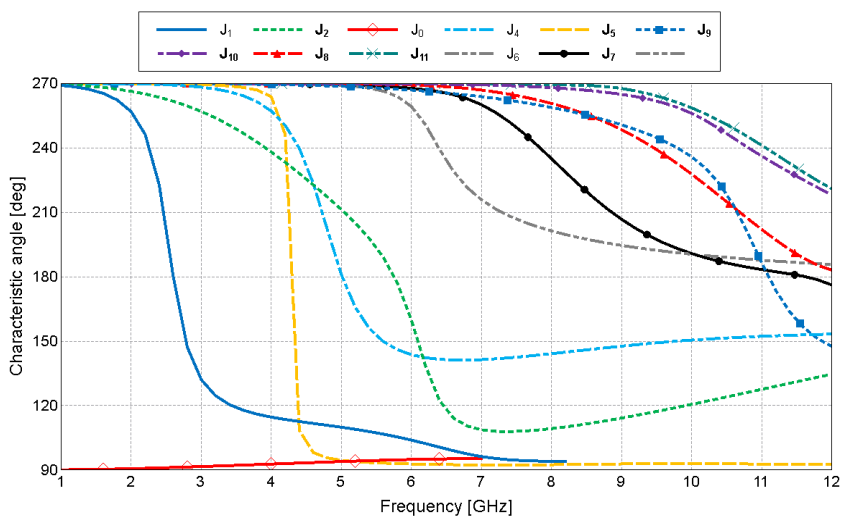

Fig. 5. Characteristic angle versus frequency for the first nine $\mathrm{CM}$ of the antenna proposed in Fig.1.

\section{Excitation of Characteristic Modes in the Proposed Structure}

Fig. 6 illustrates the contribution of each mode to the total power radiated by the antenna when using the differential feeding configuration. As observed, differential feeding facilitates the excitation of modes exhibiting vertical current distribution. Thus, mode $J_{l}$ is excited up to $7 \mathrm{GHz}$, whereas mode $J_{7}$ is excited from $7 \mathrm{GHz}$ on. A small contribution of mode $J_{8}$ is also present at higher frequencies.

Fig. 7 shows the radiated far field associated to modes $J_{l}$ and $J_{7}$ at different frequencies within the operating band. As observed, modal radiated far field varies over the frequency range, being the total radiated field a linear combination of these modal fields.

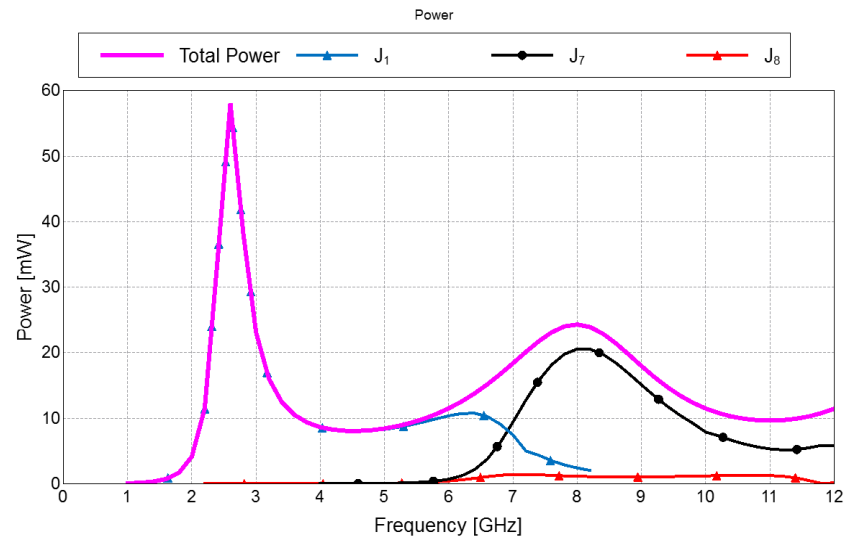

Fig. 6. Contribution of $\mathrm{CM}$ to the total radiated power of the antenna shown in Fig. 1.
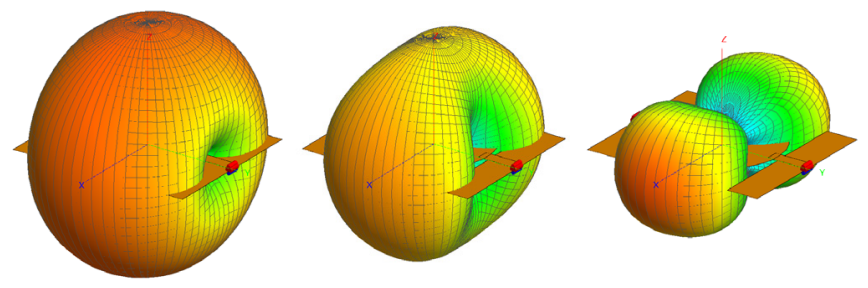

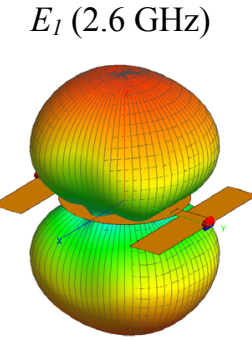

$E_{7}(7 \mathrm{GHz})$

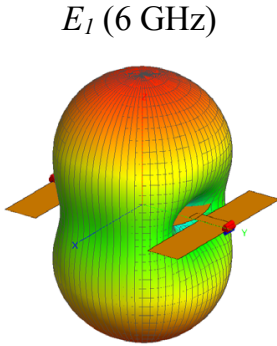

$E_{7}(9 \mathrm{GHz})$

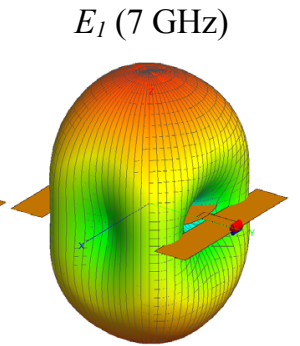

$E_{7}(11 \mathrm{GHz})$
Fig. 7. Modal radiation pattern $\left(E_{m}\right)$ associated to modes $J_{1}$ and $J_{7}$ at different frequencies.

\section{RESUlts}

In this section, the simulated results of the studied antenna structure are presented. First, the S-parameters and Active S-parameters, defined as $\mathrm{S}_{11}-\mathrm{S}_{12}$, are given. Then, the surface current distributions in $\mathrm{XY}$-cut and 3D radiation patterns are presented and compared to a monopole structure with a single feed. CST Microwave Studio 2015 was used in the simulations. 


\section{A. Simulated S-Parameters and Active S-Parameters}

Fig. 8 presents the simulated S-parameters and Active S-parameters or Differential S-parameters, as defined in [9]. Since the antenna structure is symmetrical, S-parameters of Port 2 are the same as Port 1.

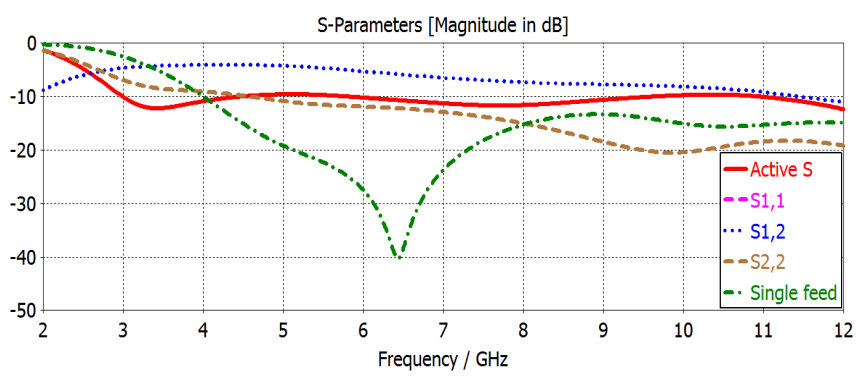

Fig. 8. Simulated Active S-parameter and S-parameters of the UWB antenna.

As it can be observed, Active S-parameters presents $-10 \mathrm{~dB}$ reflection coefficient bandwidth from $3 \mathrm{GHz}$ to more than $12 \mathrm{GHz}$, covering then UWB frequency range. As it can be noticed, the $S_{11}$ and $S_{22}$ have the same performance, since the structure is symmetrical. As expected, the $\mathrm{S}_{12}$ (equal to $\mathrm{S}_{21}$ ) is very high between the ports, but when the ports are differentially fed, the mutual coupling between the ports is interesting.

In Fig. 8 it can also be seen a curve for the single-feeding case. The curve represents the same antenna structure as that presented in Fig. 1 and with the same dimensions, but with only one rectangular ground plane and feeding line. In this case, the antenna only has one port, Port 1, and, thus, it is a typical circular monopole antenna. The single-fed monopole is a reference case to the differentially driven one, and as it can be observed, the benefit for differentially fed monopole is additional bandwidth, as well as a significant increase in the stability of the radiation pattern, as it will be seen in next section.

\section{B. Simulated Surface Current Distributions and Radiation Patterns}

In this section, the simulated surface current distributions and radiation patterns are presented. Fig. 9 presents the normalized surface current distributions of the studied differentially fed antenna at 3,5,9 and $11 \mathrm{GHz}$. As it can be observed, even higher order modes are woken, the excitation only excites radiating modes which are vertically polarized.
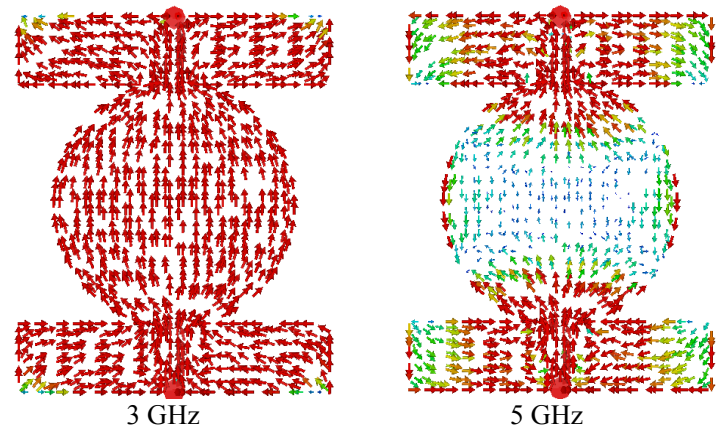
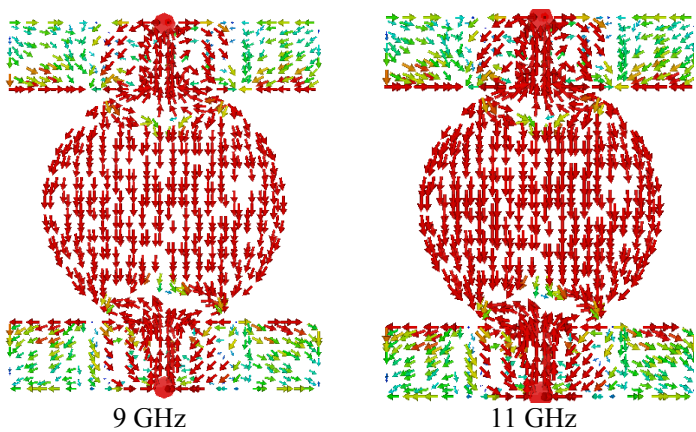

Fig. 9. Normalized total current distribution on the antenna shown in Fig. 1.

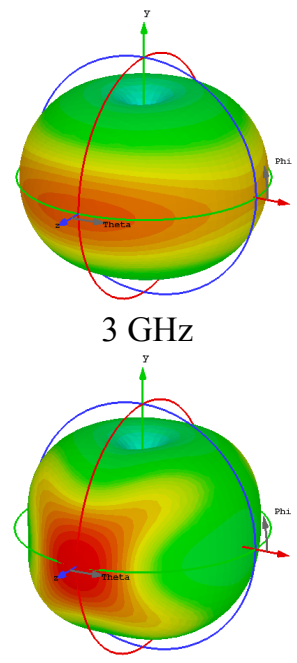

$9 \mathrm{GHz}$

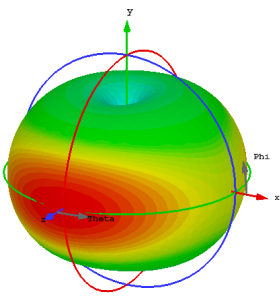

$5 \mathrm{GHz}$

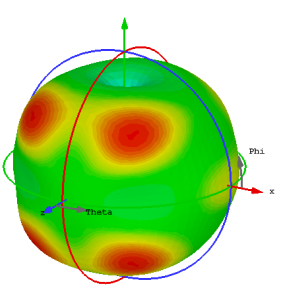

$11 \mathrm{GHz}$

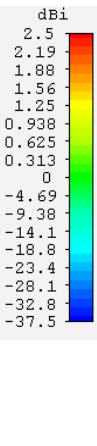

Fig. 10. Simulated 3D radiation patterns of the differentially-fed antenna shown in Fig. 1.
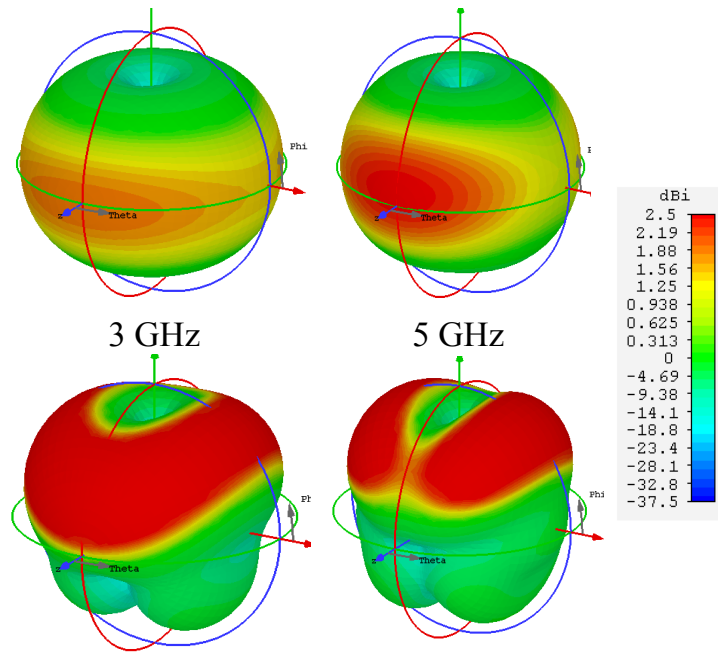

$9 \mathrm{GHz}$

$11 \mathrm{GHz}$

Fig. 11. Simulated 3D radiation patterns of a single-fed circular planar monopole antenna.

Fig. 10 presents the $3 \mathrm{D}$ radiation patterns of the differentially driven antenna studied in this paper for different frequencies within the operating frequency range. 
For the sake of comparison, Fig. 11 presents the radiation patterns of the single-fed circular monopole antenna described in Section IV.A. As it can be observed, the differentially driven antenna has more stable, symmetrical, and omni-directional radiation pattern over the studied operating bandwidth, compared to the single-fed reference monopole. Since a monopole is not a symmetrical structure, it has more directivity to the $\mathrm{Y}$ direction. The maximum gain varies between 2 and $5 \mathrm{dBi}$, depending on frequency. But for example in the direction of $X$, the gain varies only by $1 \mathrm{~dB}$ over the frequency range.

\section{CONCLUSIONS}

This paper presented simulation results for a UWB differentially driven monopole antenna using air dielectric substrate. A characteristic mode analysis has been performed in order to explain the radiating behavior of the antenna and support the use of the differential feeding to increase pattern stability. Then, it is shown that by using differential feeding, the radiation pattern is more stable and omnidirectional compared to a single fed monopole. By using differential feed, the vertical radiating modes can be excited to obtain very large bandwidth.

\section{ACKNOWLEDGMENT}

This work has been supported by Academy of Finland project Reconfigurable Antennas and Over-the-air Tests for Cognitive Radios (RAOTA), and by the Finnish Funding Agency for Innovation (Tekes), Microsoft/Oulu, Anite/Oulu, and ETS-Lindgren by project Advanced test methods of MIMO terminals in realistic usage environments (MIMOTA 2). Also by the Spanish Ministry Economy and Competitiveness under the project TEC2016-78028-C3-3-P.

\section{REFERENCES}

[1] H. Schantz, The Art and Science of Ultrawideband Antennas. Norwood, MA: Artech House, 2005.

[2] E.G. Lim, Z. Wang, C.U. Lei, Y. Wang, K.L. Man, "Ultra Wideband Antennas - Past and Present", IAENG International Journal of Computer Science, vol. 37, no.3, 2010.

[3] Z.N. Chen, M.Y.W. Chia, Broadband Planar Antennas: Design and Applications. John Wiley \& Sons, Ltd., 2006.

[4] M.C. Tang, R.W. Ziolkowski, and S. Xiao, "Compact Hyper-Band Printed Slot Antenna with Stable Radiation Properties", IEEE Trans. Antennas Propagat., vol. 62, no. 6, pp. 2962-2969, June 2014.

[5] P. Li, J. Liang, and X. Chen, "Study of Printed Elliptical/Circular Slot Antennas for Ultrawideband Applications", IEEE Trans. Antennas Propagat., vol. 54, no. 6, pp. 1670-1675, June 2006.

[6] Y. F. Liu, K. L. Lau, Q. Xue, and C. H. Chan, "Experimental Studies of Printed Wide-Slot Antenna for Wide-Band Applications", IEEE Antennas Wireless Propag. Lett., vol. 3, pp. 273-275, 2004.

[7] Z.H. Tu, W.A. Li, and Q.X. Chu, "Single-Layer Differential CPWFed Notch-Band Tapered-Slot UWB Antenna," IEEE Antennas Wireless Propag. Lett., vol. 13, pp. 1296-1299, 2014.

[8] M. Li, and K.M. Luk, "A Differential-Fed Magneto-Electric Dipole Antenna for UWB Applications," IEEE Trans. Antennas Propagat., vol. 61, no. 1, pp. 92-99, Jan. 2013.
[9] H. Huang, Y. Liu, S. Zhang, and S. Gong, "Uniplanar Differentially Driven Ultrawideband Polarization Diversity Antenna With BandNotched Characteristics," IEEE Antennas Wireless Propag. Lett., vol. 14, pp. 563-566, 2015.

[10] M. Sonkki, E. Antonino-Daviu, N. Mohamed Mohamed-Hicho, M. Ferrrando-Bataller, E. Salonen, "UWB Planar Monopole Antenna with Differential Feed”, 27th Annual IEEE International Symposium of Personal, Indoor and Mobile Radio Communications (PIMRC'16), 4-7 September 2016, Valencia, Spain.

[11] E. Antonino-Daviu, N. Mohamed Mohamed-Hicho, , M. CabedoFabrés and M. Ferrando-Bataller, "Modal Pattern Stability Factor as a Figure of Merit for Characteristic Modes", Electronics Letters, 2 (52), pp. $1658-1659$, Sept. 2016. 